\title{
Identification of Potential Key lncRNAs and Genes Associated with Aging Based on Microarray Data of Adipocytes from Mice
}

\author{
Yi Yang, Zongyan Teng, Songyan Meng, and Weigang Yu \\ Department of Geriatrics, The Second Affiliated Hospital of Harbin Medical University, Harbin, Heilongjiang 150086, China \\ Correspondence should be addressed to Yi Yang; yangyi_yiu@163.com
}

Received 22 July 2016; Revised 19 October 2016; Accepted 1 November 2016

Academic Editor: Terri L. Young

Copyright ( 2016 Yi Yang et al. This is an open access article distributed under the Creative Commons Attribution License, which permits unrestricted use, distribution, and reproduction in any medium, provided the original work is properly cited.

\begin{abstract}
Objective. This study aimed to screen potential crucial lncRNAs and genes involved in aging. Methods. The data of 9 peripheral white adipocytes, respectively, taken from male C57BL/6J mice (6 months, 14 months, and 18 months of age) in GSE25905 were used in this study. Differentially time series expressed lncRNA genes (DE-lncRNAs) and mRNA genes (DEGs) were identified. After cluster analysis of lncRNAs expression pattern, target genes of DE-lncRNAs were predicted from the DEGs, and functional analysis for target genes was conducted. Results. A total of 8301 time series-related DEGs and 43 time series-related DE-lncRNAs were identified. Among them, 41 DE-lncRNAs targeted 1880 DEGs. The DEGs positively regulated by DE-lncRNAs were mainly related to the development of blood vessel and the pathways of cholesterol biosynthesis and elastic fibre formation. Furthermore, the DEGs negatively regulated by DE-lncRNAs were correlated with protein metabolism. Conclusion. These DE-lncRNAs and DEGs are potentially involved in the process of aging.
\end{abstract}

\section{Introduction}

Aging is an elevated risk of common diseases, including obesity, hypertension, atherosclerosis, and diabetes [1-3]. Currently, about 800 million people are at least 60 years old, which accounts for about $11 \%$ of the world's population, and aging population is estimated to increase to more than 2 billion by 2050 [4]. Aging is closely related to damaged adipogenesis in various fat depots in humans $[5,6]$. White adipose tissue (WAT) is considered as an important regulator for multiple physiological processes and highly linked to the development of multiple morbidities [7-9]. Therefore, it is significant and urgent to reveal the relationships of aging and adipose, which is very important for understanding the diseases in the elderly.

Previous studies have discovered a set of genes that are implicated in the aging process in an adipose depotdependent manner. For example, age-related increase in $I L$ 6 (interleukin 6), which was related to stress responses and cellular senescence, was observed in a fat depot-dependent manner [5]. Sirt1 (sirtuin 1) and SOD2 (superoxide dismutase 2 ), which were correlated with mitochondrial aging, were significantly decreased in aging epididymal adipocytes [10].
Furthermore, the expression of MMP-3 (matrix metallopeptidase 3) was increased in mouse subcutaneous fat cells and human skin fibroblasts with aging [11, 12]. Additionally, decreased expression in PPAR $\gamma$ (peroxisome proliferatoractivated receptor gamma) through declining fat mass has been observed in monkey subcutaneous whole fat tissue [13]. In addition to these genes mentioned above, roles of long noncoding RNAs (lncRNAs) in age-related diseases have attracted more attention recently $[14,15]$. LncRNAs are defined as the largest transcript class in human genome longer than $200 \mathrm{bp}$ that lack protein-coding potential $[16,17]$. In aging murine aortas, mitochondrial lncRNA ASncmtRNA-2 is induced by replicative senescence [18]. Abnormal expression of the telomeric repeat-containing RNA lncRNA TERRA is responsible for premature senescence and aging through controlling telomere elongation [19]. In spite of much effort, the lncRNAs with known functions involved in aging remain rare.

Microarray technology has been widely used in molecular studies of human diseases [20,21]. Based on an age-related gene expression profile GSE25905, Liu et al. have found high expression of genes involved in inflammatory response and low adipose-specific gene expression in bone marrow 
adipocytes, and age has a greater influence on gene expression in epididymal adipocytes than bone marrow adipocytes [22]. However, the effect of aging on expression of lncRNAs is still elusive.

In the current study, to investigate the expression variation and functional roles of lncRNAs in aging, the microarray data deposited by Liu et al. [22] were used to identify the differentially time series expressed lncRNA genes (DElncRNAs) and differentially time series expressed mRNA genes (DEGs) in the process of aging. Additionally, DEGs targeted by DE-lncRNAs and their functions were analyzed. The results may provide new information for the molecular investigation of aging and a deeper insight into aging.

\section{Methods and Materials}

2.1. Tissue Samples and Data Acquisition. The gene expression profile GSE25905 [22] was downloaded from the Gene Expression Omnibus (GEO, http://www.ncbi.nlm.nih .gov/geo/) database in National Center for Biotechnology Information (NCBI). The microarray data were produced on GPL6246 platform ([MoGene-1_0-st] Affymetrix Mouse Gene 1.0 ST Array, Affymetrix, CA, USA). This dataset contained 9 bone marrow adipocyte samples and 9 peripheral white adipocytes, respectively, taken from male C57BL/6J mice (6 months, 14 months, and 18 months of age), with three replicates at each age point. In this study, only gene microarray data of peripheral white adipocytes were used for further analysis.

2.2. Data Preprocessing. The gene expression profile of GSE25905 was preprocessed by the Robust Microarray Analysis (RMA) algorithm [23]. The Affy package (available at http://master.bioconductor.org/packages/release/bioc/html/ affy.html) [24] of R. Probe IDs in CEL document was translated to corresponding gene symbols. If one gene symbol was matched by multiple probe IDs, the mean expression value was selected as the expression level of this gene.

2.3. Identification of DE-lncRNAs and DEGs. Based on annotation information of lncRNAs in GENCODE (http://www .gencodegenes.org/) [25] and the array platform GPL6246, expression data of lncRNAs were obtained. Afterwards, the BETR (Bayesian Estimation of Temporal Regulation) algorithm in the BETR package (http://betterpackages.com/) [26] was applied to identify DE-lncRNAs and DEGs at the three time points, and this algorithm calculated the probability of differential expression for each gene. The probability $>0.9$ was set as the cut-off criterion.

2.4. Cluster Analysis of DE-lncRNAs Expression Pattern. Hierarchical clustering is an analytical tool applied to discover the closest associations between gene profiles and specimens under evaluation $[27,28]$. In our study, the $\mathrm{BHC}$ (Bayesian Hierarchical Clustering) package (http://master.bioconductor.org/packages/release/bioc/html/BHC.html) [29] of $\mathrm{R}$ was utilized to perform clustering of DE-lncRNAs and construct the cluster heat map of DE-lncRNAs and samples.
2.5. Prediction of DE-lncRNA Target Genes. Pearson correlation coefficient (PCC) [30] was used to calculate the expression similarity of DE-lncRNAs and DEGs at different time points. For each pair of DE-lncRNA and DEGs, significant correlation pairs with $|\mathrm{PCC}|>0.95$ and $p$ value $<0.05$ were used to construct the DE-lncRNA/DEG regulatory network which was then visualized by Cytoscape (http://js.cytoscape.org/) [31].

Furthermore, DE-lncRNA target genes that were known to be associated with aging were identified based on the information in AGEMAP (Atlas of Gene Expression in Mouse Aging Project), which is a gene expression database for aging in mice (http://cmgm.stanford.edu/ kimlab/aging_mouse) [32]. Subsequently, the regulatory network of DE-lncRNAs and the known aging-related targets was visualized by Cytoscape.

2.6. Functional Analysis. Gene Ontology (GO) functional and pathway enrichment analyses for genes positively and negatively regulated by DE-lncRNAs were carried out using TargetMine (http://targetmine.mizuguchilab.org/) [33]. The $p$ value of each GO and pathway term was adjusted by the Holm-Bonferroni method [34], and adjusted $p$ value $<$ 0.05 was considered statistically significant. Additionally, the pathway network was constructed using Cytoscape.

\section{Results}

3.1. Identified DE-lncRNAs and DEGs. Based on the annotation information in GENCODE and GPL6246 platform, a total of 203 probes were annotated as lncRNA genes, and 20564 probes were annotated as mRNA genes. With the cut-off of probability $>0.9,8301$ time series DEGs and 43 DE-lncRNAs were identified in peripheral white adipocyte samples.

3.2. Clusters of DE-lncRNAs Expression Pattern. To further explore the changes of the DE-lncRNAs expression levels at the three time points in peripheral white adipocytes, the cluster analysis was conducted. The samples at different time points were distinguished by DE-lncRNAs. The expression values of nearly half of DE-lncRNAs showed an uptrend in 6-14 months and a downtrend in 14-18 months (e.g., ENSMUSG00000086859 and ENSMUSG00000061510); a set of DE-lncRNAs were expressed in a decline trend (e.g., ENSMUSG00000087540 and ENSMUSG00000032048); and a small fraction of DE-lncRNAs were expressed in a rising trend (e.g., ENSMUSG00000066057) (Figure 1(a)).

According to the results of clustering analysis, $41 \mathrm{DE}$ lncRNAs were divided into 11 clusters (Table 1). It was clearly observed that, with the increase of age in mice, DE-lncRNAs in clusters 7 and 10 were expressed in a rising trend, whereas DE-lncRNAs in clusters 2, 4, and 8 were expressed in a decline trend. Clusters 5 and 11 showed an uptrend in 6-14 months and a downtrend in 14-18 months (Figure 1(b)).

3.3. DEGs Targeted by DE-lncRNAs. LncRNAs have critical roles in the transcriptional regulation via modulating the gene expressions. To further investigate the regulatory 

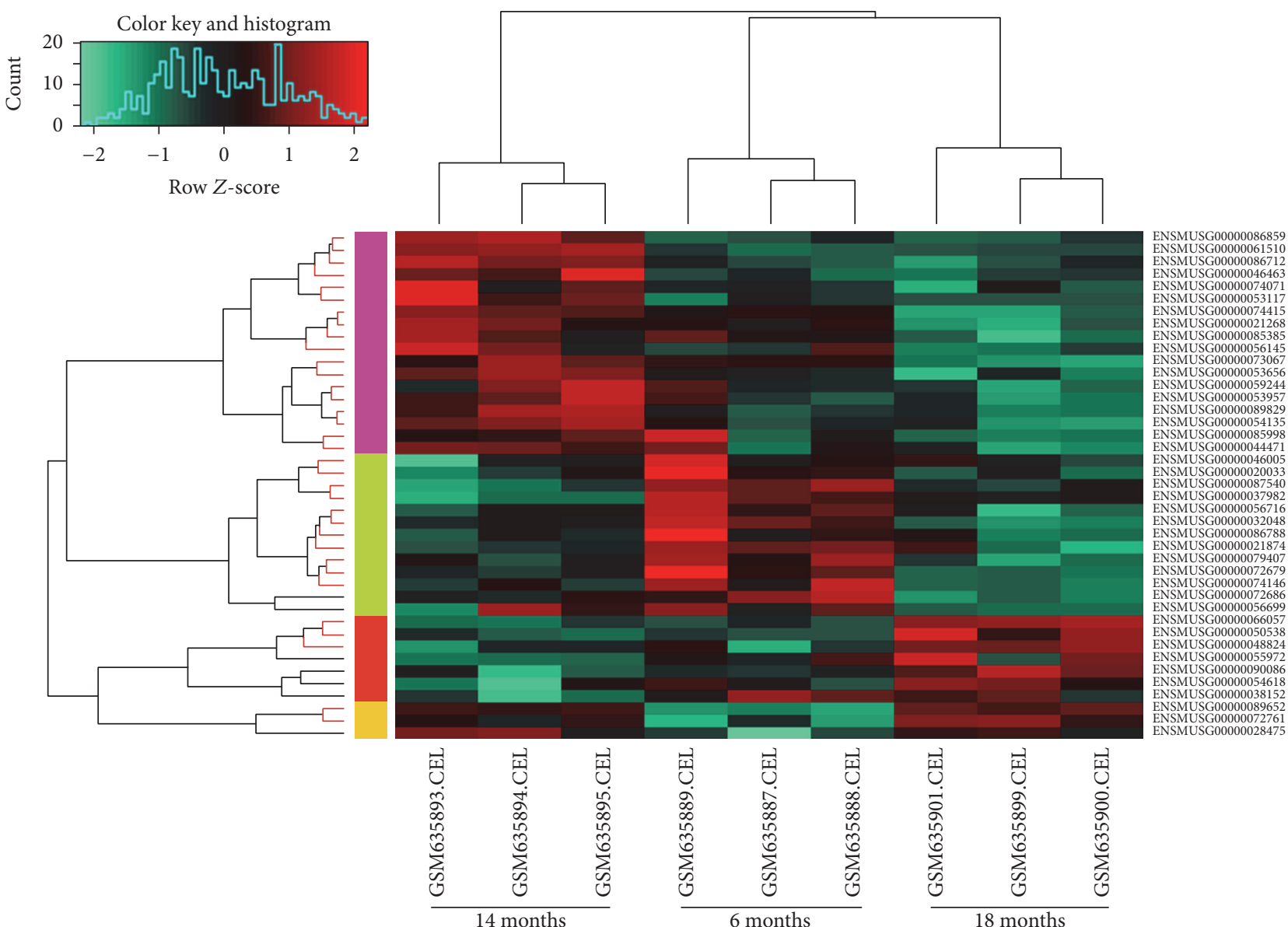

(a)

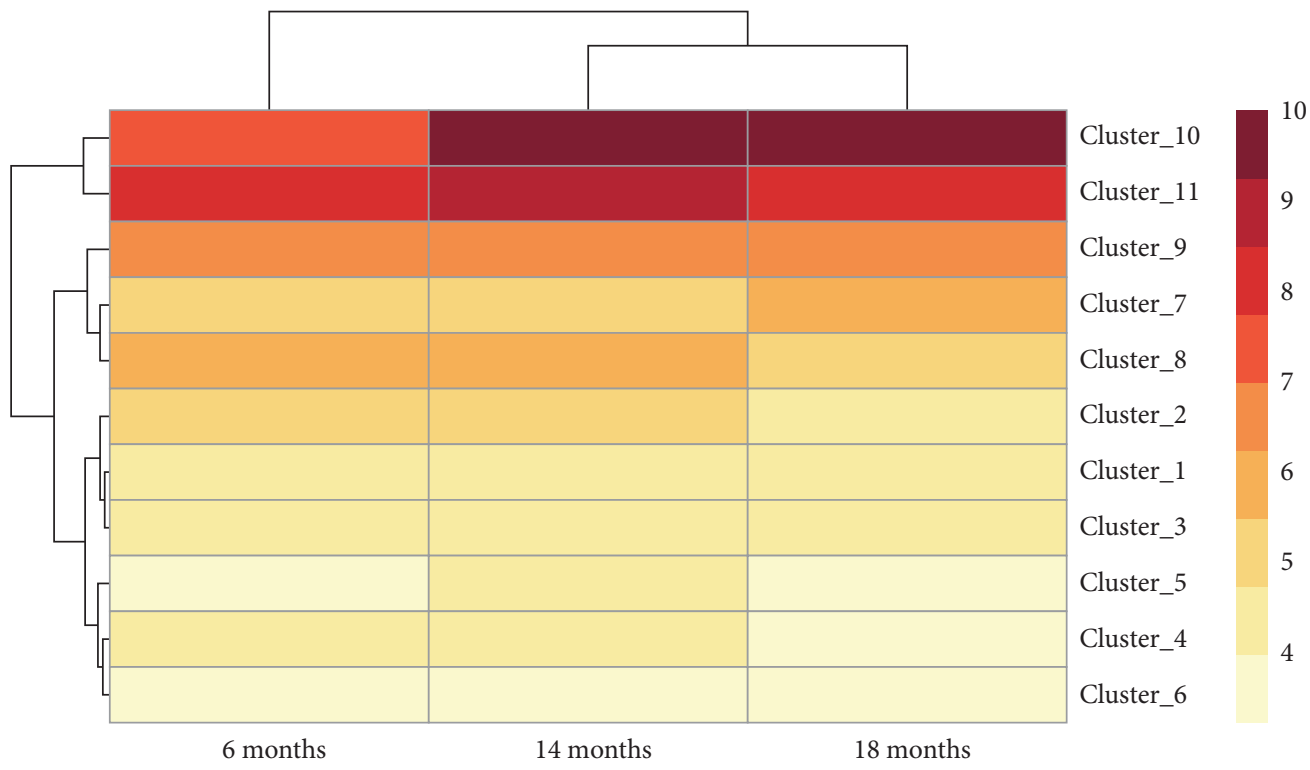

(b)

FIGURE 1: Heat maps of differentially expressed lncRNA genes. (a) Heat map of differentially expressed lncRNA genes in peripheral white adipocytes samples from male C57BL/6J mice being 6 months, 14 months, and 18 months of age. Each row represents a single gene; each column represents a sample. The gradual color change from red to green represents the changing process of expression level from upregulation to downregulation. (b) Heat map of clusters of differentially expressed lncRNA genes at 6, 14, and 18 months. Each row represents a cluster; each column represents a time point. LncRNA, long noncoding RNA. 
TABLE 1: Differentially time-series expressed long noncoding RNA genes in each cluster.

\begin{tabular}{lcr}
\hline $\begin{array}{l}\text { Cluster } \\
\text { number }\end{array}$ & Count & Differentially time-series expressed long noncoding RNA gene ID \\
\hline 1 & 3 & ENSMUSG00000079407, ENSMUSG00000072686, ENSMUSG00000032048 \\
2 & 2 & ENSMUSG00000072679, ENSMUSG00000073067 \\
3 & 8 & $\begin{array}{c}\text { ENSMUSG00000056145, ENSMUSG00000090086, ENSMUSG00000074071, ENSMUSG00000046463, } \\
\text { ENSMUSG00000055972, ENSMUSG00000053957, ENSMUSG00000048824, ENSMUSG00000053117 }\end{array}$ \\
4 & 6 & ENSMUSG00000046005, ENSMUSG00000021874, ENSMUSG00000056699, ENSMUSG00000059244, \\
5 & 1 & ENSMUSG00000056716, ENSMUSG00000086788 \\
6 & 3 & ENSMUSG00000087540, ENSMUSG00000074146, ENSMUSG00000020033 \\
7 & 4 & ENSMUSG00000050538, ENSMUSG00000054618, ENSMUSG00000038152, ENSMUSG00000089829 \\
8 & 6 & ENSMUSG00000054135, ENSMUSG0000005365, ENSMUSG00000086859, ENSMUSG00000086712, \\
9 & 6 & ENSMUSG00000074415, ENSMUSG00000021268 \\
10 & 1 & ENSMUSG00000085998, ENSMUSG00000072761 \\
11 & 1 & ENSMUSG00000089652 \\
\hline
\end{tabular}

TABLE 2: Results of Gene Ontology functional and pathways enrichment analyses for genes positively regulated by differentially time-series expressed lncRNAs.

\begin{tabular}{|c|c|c|c|c|}
\hline Category & Term & Adjust $p$ value & Gene count & Genes \\
\hline GO-BP & $\begin{array}{l}\text { Vasculature development } \\
\text { [GO:0001944] }\end{array}$ & $1.33 E-08$ & 65 & $\begin{array}{c}\text { Adamts1, Cdh5, Ctsh, Cxcr3, Dhcr7, Edn1, Efnal, Efnb2, } \\
\text { Notch3, Pdgfrb, and so on }\end{array}$ \\
\hline GO-BP & $\begin{array}{l}\text { Blood vessel development } \\
\text { [GO:0001568] }\end{array}$ & $1.34 E-07$ & 60 & $\begin{array}{c}\text { Adamts1, Cdh5, Ctsh, Cxcr3, Dhcr7, Edn1, Efnal, Efnb2, } \\
\text { Notch3, Pdgfrb, and so on }\end{array}$ \\
\hline GO-BP & $\begin{array}{l}\text { Regulation of locomotion } \\
\text { [GO:0040012] }\end{array}$ & $2.45 E-05$ & 51 & $\begin{array}{c}\text { Dab2, Ifitm3, Ill6, Il33, Irs2, Megf8, Myolf, Pdgfrb, } \\
\text { Pecam1, Pkn1, and so on }\end{array}$ \\
\hline GO-BP & $\begin{array}{l}\text { Blood vessel } \\
\text { morphogenesis } \\
\text { [GO:0048514] }\end{array}$ & $2.55 E-05$ & 49 & $\begin{array}{c}\text { Adamts1, Aqp1, C3, Ccr2, Edn1, Efna1, Efnb2, Egfl7, Elk3, } \\
\text { Ephb4, and so on }\end{array}$ \\
\hline GO-BP & $\begin{array}{l}\text { Regulation of cellular } \\
\text { component movement } \\
\text { [GO:0051270] }\end{array}$ & $3.92 E-05$ & 50 & $\begin{array}{c}\text { Ccl21a, Dab2, Ddr2, Dpep1, Efnal, Nup155, Pde4d, Pdgfra, } \\
\text { Pdgfrb, Pecam1, and so on }\end{array}$ \\
\hline GO-CC & Cell surface [GO:0009986] & $2.46 E-05$ & 53 & $\begin{array}{c}\text { Ackr3, Alcam, Cd200r1, Cd3e, Dpp4, Enpp1, Flt3l, Heg1, } \\
\text { Ifitm3, Il2rb, and so on }\end{array}$ \\
\hline $\mathrm{GO}-\mathrm{CC}$ & $\begin{array}{l}\text { Plasma membrane } \\
\text { [GO:0005886] }\end{array}$ & $9.39 E-05$ & 158 & $\begin{array}{c}\text { Ano1, Antxr1, Aqp1, Capn3, Ccr2, Itga5, Itm2c, Kcnab1, } \\
\text { Kcnn3, Kcnt2, and so on }\end{array}$ \\
\hline GO-CC & $\begin{array}{l}\text { Cell periphery } \\
\text { [GO:0071944] }\end{array}$ & $2.59 E-04$ & 163 & $\begin{array}{c}\text { Antxr1, Aqp1, Bcas3, Capn3, Eps15l1, Ezr, Krt19, Lime1, } \\
\text { Ntn4, P2rx4, and so on }\end{array}$ \\
\hline $\mathrm{GO}-\mathrm{CC}$ & $\begin{array}{l}\text { Side of membrane } \\
\text { [GO:0098552] }\end{array}$ & 0.015056 & 33 & $\begin{array}{c}\text { Alcam, Ano1, Cd74, Ikbkb, Il2rb, Itga5, Kdr, Ly6a, Ly6c1, } \\
\text { Pkp4, and so on }\end{array}$ \\
\hline $\mathrm{GO}-\mathrm{CC}$ & $\begin{array}{l}\text { Plasma membrane part } \\
\text { [GO:0044459] }\end{array}$ & 0.021127 & 101 & $\begin{array}{c}\text { Klri1, Lime1, Ly6a, Npc1, P2rx4, Sema6a, Sept2, Tspan15, } \\
\text { Upk1b, Zdhhc2, and so on }\end{array}$ \\
\hline REACT_208531 & Cholesterol biosynthesis & $4.00 E-03$ & 9 & Cyp51, Dhcr7, Fdps, Hsd17b7, Idi1, Lss, Mvk, Sc5d, Sqle \\
\hline REACT_198996 & Elastic fibre formation & $5.10 E-03$ & 11 & $\begin{array}{c}\text { Efemp1, Efemp2, Fbln1, Fbln2, Fbln5, Furin, Itga5, Loxl1, } \\
\text { Ltbp1, Mfap3, and so on }\end{array}$ \\
\hline
\end{tabular}

The GO-BP terms in the table are the top 5 ones with a higher adjusted $p$ value. DE-lncRNA, differentially time-series expressed long noncoding RNA gene; GO, Gene Ontology; BP, biological process; CC, cellular component. "REACT" terms are the pathway terms.

functions of DE-lncRNAs, the DEGs regulated by DElncRNAs were analyzed by the PCC algorithm. Based on the cut-off criteria, 2313 regulatory relationships between DE-lncRNAs and DEGs were obtained (see Supplementary Material available online at http://dx.doi.org/10.1155/2016/
9181702). The constructed regulatory network consisted of 41 DE-lncRNAs and 1880 DEGs. The DE-lncRNAs ENSMUSG00000066057, ENSMUSG00000086859, and ENSMUSG00000061510 modulated more DEGs than others. ENSMUSG00000086859 targeted genes like Efnal, Fbln1, 


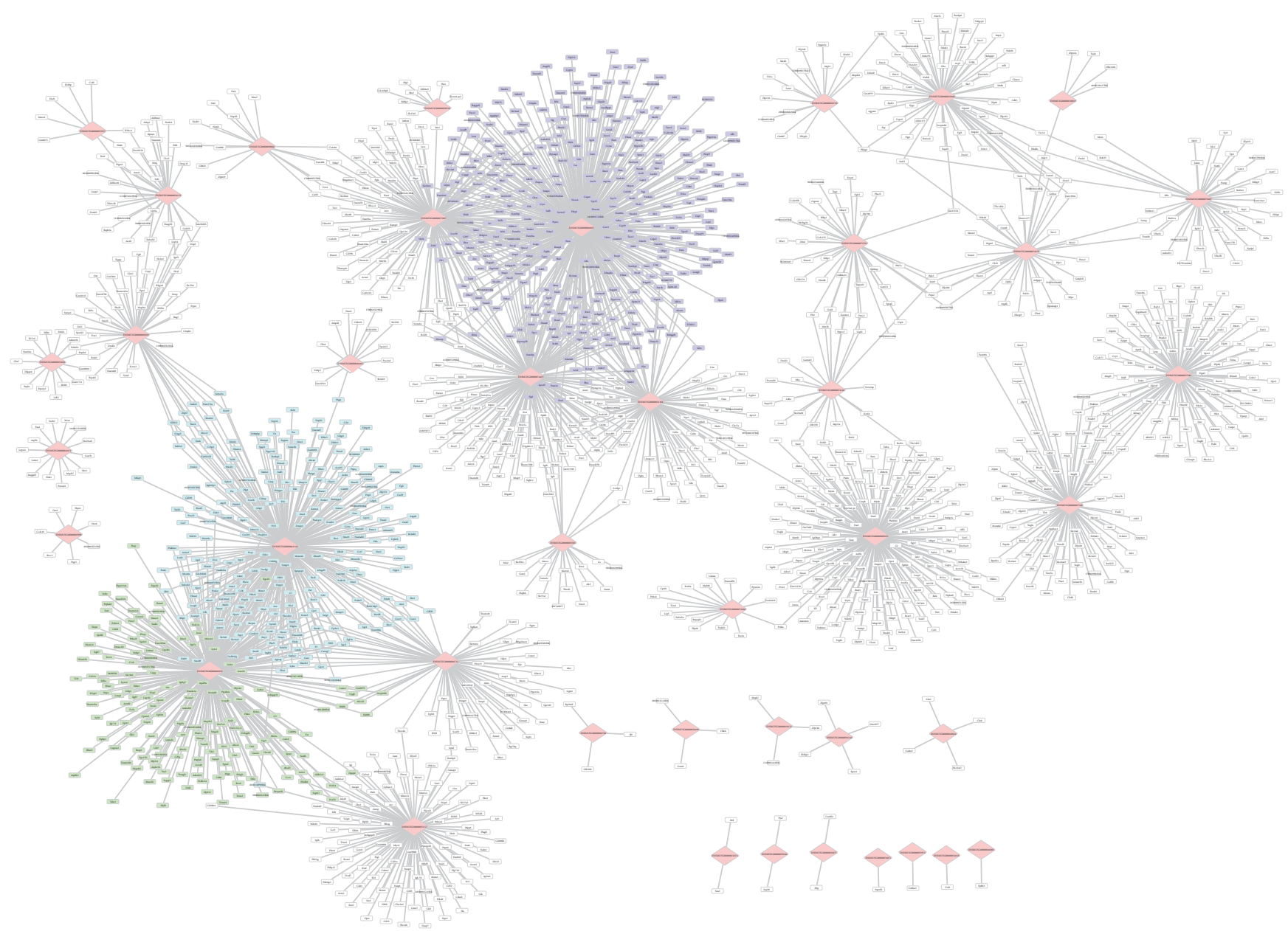

FIGURE 2: The regulatory network of 41 differentially expressed lncRNA genes and their target mRNA genes. The diamonds represent lncRNA gene IDs, and rectangles represent mRNA genes. The purple nodes represent the target genes of ENSMUSG00000066057; the blue nodes represent the target genes of ENSMUSG00000061510; the green nodes represent the target genes of ENSMUSG00000086859. LncRNA, long noncoding RNA.

and Fbln2. ENSMUSG00000066057 regulated the DEGs, such as CYP51, FDPS, and Eif2s1 (Figure 2). The expression changes over time of ENSMUSG00000086859 and ENSMUSG00000066057, as well as some targets of them, were shown in Figure 3.

Based on the AGEMAP database, a total of $51 \mathrm{DE}-$ lncRNA target genes had been discovered to be correlated with aging in mice. There were $16 \mathrm{DE}-\operatorname{lncRNAs}$ that regulated these genes, and 61 regulatory relationships were included in the network. Both ENSMUSG00000086859 and ENSMUSG00000061510 targeted Slc16a2 and Ifitm3; ENSMUSG00000066057 regulated aging-related DEGs like Wdr1 (Figure 4(a)).

3.4. Enrichment Analysis of DE-lncRNA Targets. To further reveal the potential functions mediated by DE-lncRNAs, the GO and pathway enrichment analyses of DE-lncRNA targets were performed, respectively. The DEGs positively regulated by DE-lncRNAs (e.g., Efnal and $E f n b 2$ ) were mainly enriched in a set of biology processes about the development of blood vessel, such as vasculature development and blood vessel morphogenesis (Table 2). A series of positively regulated target genes were significantly enriched in the pathways of cholesterol biosynthesis (e.g., Cyp51 and Fdps) and elastic fibre formation (e.g., Fbln1, Fbln2, and Fbln5) (Table 2).

Furthermore, the negatively regulated target genes of DElncRNAs were mainly enriched in a set of biology processes, such as metabolic process (e.g., Abi3 and Acaca) and mitochondrion organization (e.g., Acaa2 and Bnip3), as well as pathways like metabolism of proteins (e.g., Eif2sl, Eif2s $3 x$, and Eif3b) (Table 3).

Additionally, the pathway of metabolism of proteins was predicted to interact with five other pathways, such as posttranslational protein modification and asparagine $\mathrm{N}$-linked glycosylation (Figure 4(b)).

\section{Discussion}

The increased occurrence of age-related diseases, such as cancers, chronic inflammatory, and neurodegenerative diseases, becomes a burden on health care provision in the developed 


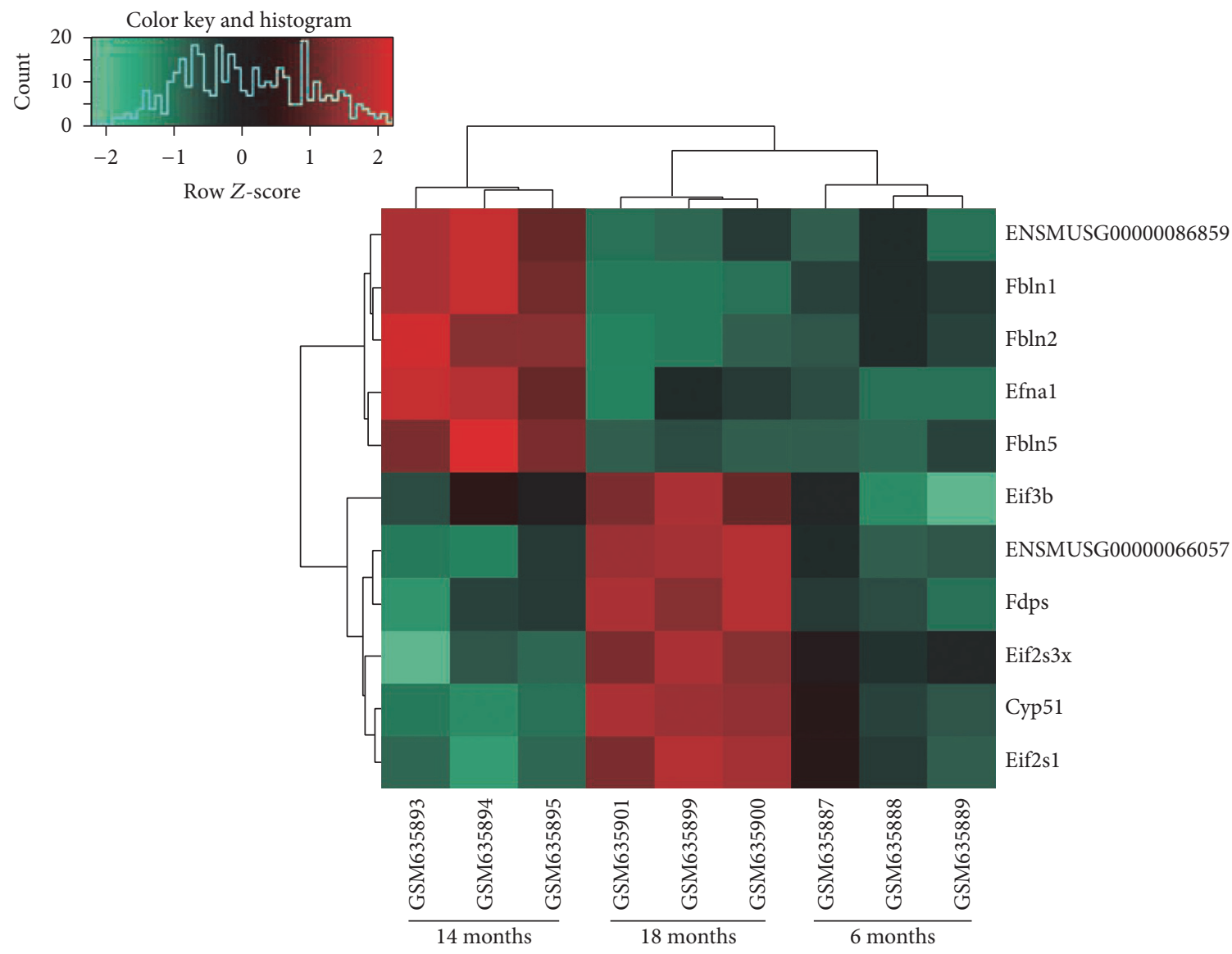

FIGURE 3: Heat map showing the expression changes over time of ENSMUSG00000086859 and ENSMUSG00000066057, as well as some targets of them. Each row represents a single gene or lncRNA; each column represents a sample. The gradual color change from red to green represents the changing process of expression level from upregulation to downregulation. LncRNA, long noncoding RNA.

and developing countries [35]. In this study, gene expression profile GSE25905 was downloaded and analyzed using bioinformatics methods to explore the potential mechanisms of aging. A total of 8301 time series DEGs and 43 time series DE-lncRNAs were identified in peripheral white adipocyte samples. In the DE-lncRNAs/DEGs regulatory network, the DE-lncRNAs ENSMUSG00000066057, ENSMUSG00000086859, and ENSMUSG00000061510 regulated multiple DEGs. The DEGs positively regulated by DElncRNAs were mainly enriched in the functions about the development of blood vessel (e.g., Efnal and Efnb2), as well as the pathways of cholesterol biosynthesis (e.g., Cyp51 and $F d p s$ ) and elastic fibre formation (e.g., Fbln1, Fbln2, and Fbln5).

The function of blood vessel development was significantly enriched by a set of DE-lncRNA genes, such as Efnal and Efnb2. During aging, angiogenesis is delayed, and capillary density as well as newly deposited collagen is decreased [36]. Cardiovascular structure and function are altered during aging, with elongated and stiffer aorta, as well as changed arterial baroreflex [37]. Both Efnal and $E f n b 2$ encode members of the ephrin family, which mediates developmental events [38]. It has been confirmed that the balance of alternatively expressed isoforms in Efnal is disrupted in peripheral blood leukocytes of human population with advancing age [39]. Furthermore, the expression of $E f n b 2$ is significantly decreased during the aging of the rat retina [40]. In this study, Efnal was predicted to be regulated by the DE-lncRNA ENSMUSG00000086859 (gene name: 2810008D09Rik). There is no study that reports the role of ENSMUSG00000086859 in aging so far. Collectively, we speculate that ENSMUSG00000086859 may play key roles in aging through genes related to blood vessel development (e.g., Efnal).

In this study, several other DEGs positively regulated by DE-lncRNAs (e.g., $F b \ln 1, F b \ln 2$, and $F b \ln 5$ ) were significantly enriched in the pathway of elastic fibre formation. During cutaneous aging, elastic fibres exhibited disintegration and appeared to be loose [41]. All of Fbln1, Fbln2, and Fbln 5 encode a secreted glycoprotein that is incorporated into a fibrillar extracellular matrix [42]. During aging, the balance between proteases and their inhibitors involved in extracellular matrix formation is destroyed [43]. In this study, $F b \ln 1$ and $F b \ln 2$ were discovered to be an age-regulated gene and regulated by the DE-lncRNA ENSMUSG00000086859 (gene name: 2810008D09Rik). The association of Fbln2 

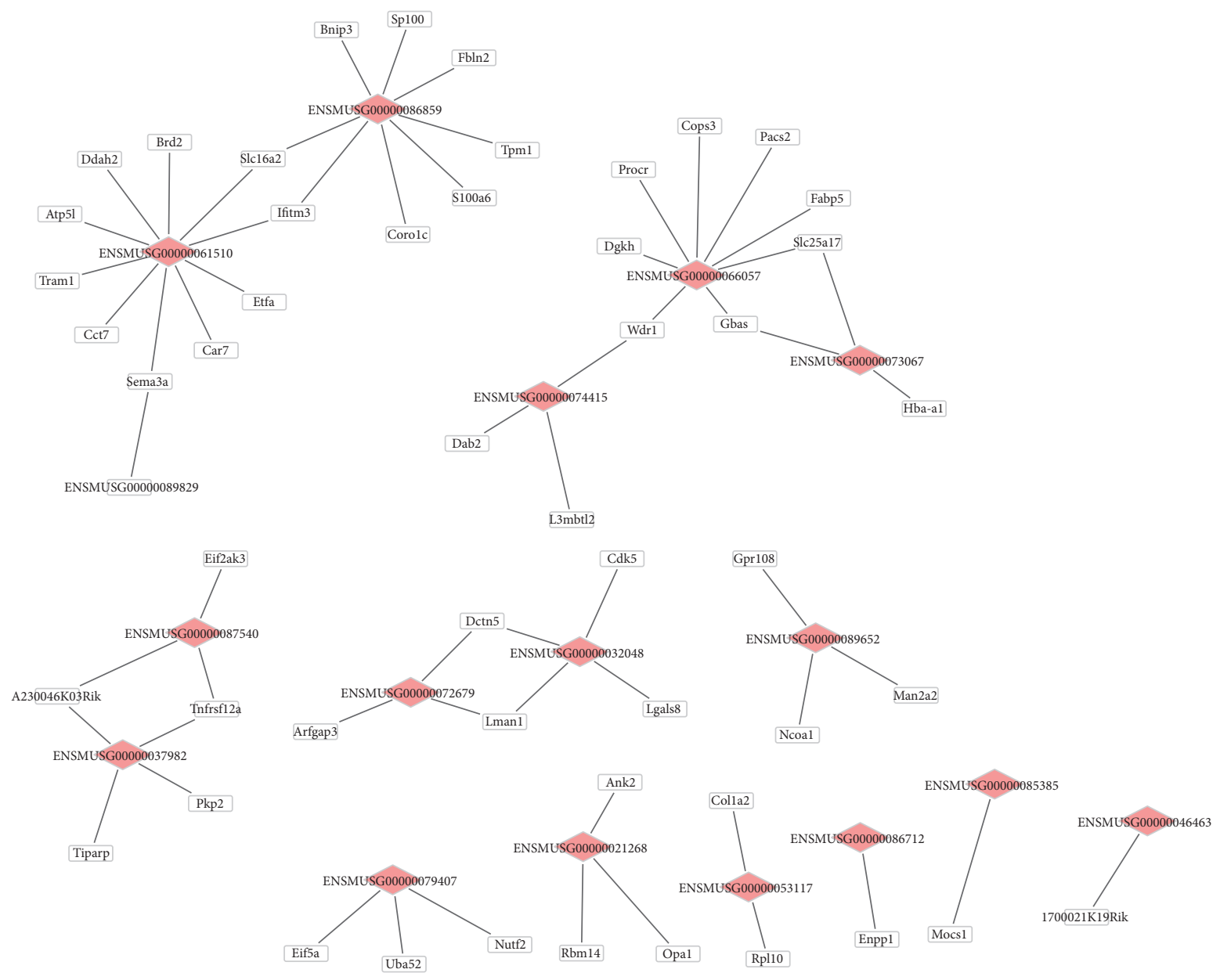

(a)

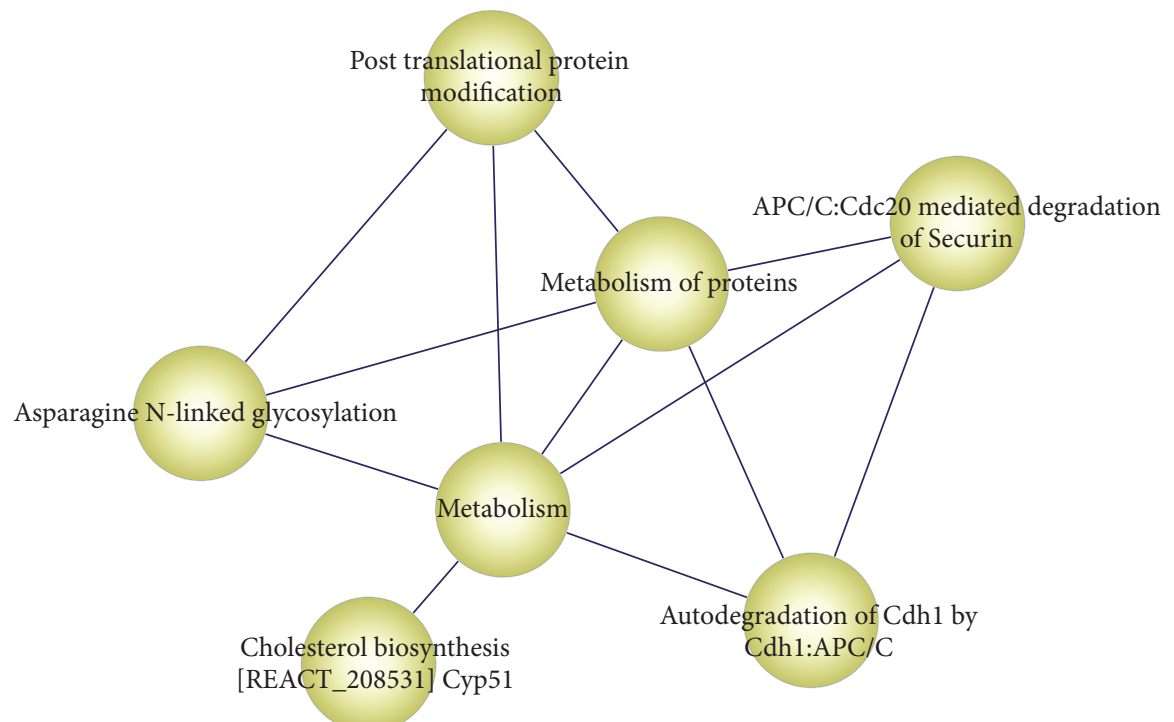

(b)

FIGURE 4: Networks of genes and pathways. (a) The regulatory network of differentially expressed lncRNA genes and age-related target genes. The diamonds represent lncRNA gene IDs, and rectangles represent mRNA genes. (b) The network of pathways that are enriched by target genes. Nodes represent pathways, and lines indicate that there are common genes related to the two pathways. The larger the thickness of line is, the more the common genes are. LncRNA, long noncoding RNA. 
TABLE 3: Results of Gene Ontology functional and pathways enrichment analyses for genes negatively regulated by differentially time-series expressed lncRNAs.

\begin{tabular}{|c|c|c|c|c|}
\hline Category & Term & Adjust $p$ value & Gene count & Genes \\
\hline GO-BP & $\begin{array}{l}\text { Metabolic process } \\
\text { [GO:0008152] }\end{array}$ & $8.05 E-10$ & 380 & $\begin{array}{c}\text { Abi3, Acaca, Acadvl, Brd2, Capn15, Cxcl9, D2hgdh, Dagla, } \\
\text { Gemin5, Gfml, and so on }\end{array}$ \\
\hline GO-BP & $\begin{array}{c}\text { Organic substance } \\
\text { metabolic process } \\
\text { [GO:0071704] }\end{array}$ & $5.74 E-09$ & 360 & $\begin{array}{c}\text { Acox1, Acsm3, Capn15, Casp2, Ddx20, Decr1, Dennd3, } \\
\text { Frzb, Fxr1, Gadd45a, and so on }\end{array}$ \\
\hline GO-BP & $\begin{array}{l}\text { Primary metabolic process } \\
\text { [GO:0044238] }\end{array}$ & $3.62 E-08$ & 343 & $\begin{array}{c}\text { Acadvl, Acot7, Babam1, Bach1, Eif3c, Ell, Gbp2, Gclm } \\
\text { Mtdh, Nabp1, and so on }\end{array}$ \\
\hline GO-BP & $\begin{array}{l}\text { Cellular metabolic process } \\
\text { [GO:0044237] }\end{array}$ & $5.03 E-08$ & 344 & $\begin{array}{c}\text { Acadvl, Acot7, Babam1, Bach1, Eif3c, Ell, Gbp2, Gclm } \\
\text { Mtdh, Nabp1, and so on }\end{array}$ \\
\hline GO-BP & $\begin{array}{c}\text { Mitochondrion } \\
\text { organization [GO:0007005] }\end{array}$ & $2.30 E-04$ & 32 & $\begin{array}{c}\text { Acaa2, Bnip3, Cln8, Dap3, March5, Mrpl44, Mtch2, } \\
\text { Mtfr2, Ptcd2, Slc22a5, and so on }\end{array}$ \\
\hline $\mathrm{GO}-\mathrm{CC}$ & Intracellular [GO:0005622] & $2.01 E-16$ & 478 & $\begin{array}{c}\text { Abi3, Acaa2, Acyp2, Adap2, Capn15, Ggct, Ggh, Itgblbp1, } \\
\text { Jak2, Katna1, and so on }\end{array}$ \\
\hline $\mathrm{GO}-\mathrm{CC}$ & $\begin{array}{l}\text { Intracellular part } \\
\text { [GO:0044424] }\end{array}$ & $3.17 E-16$ & 475 & $\begin{array}{c}\text { Abi3, Acaa2, Acyp2, Adap2, Capn15, Ggct, Ggh, Itgblbp1, } \\
\text { Jak2, Katna1, and so on }\end{array}$ \\
\hline $\mathrm{GO}-\mathrm{CC}$ & Cell [GO:0005623] & $4.05 E-15$ & 526 & $\begin{array}{c}\text { Atp5l, B4galt1, Dusp8, Dynll2, Exoc4, Faf1, Hint2, Hmoxl, } \\
\text { Rtn2, S100al1, and so on }\end{array}$ \\
\hline $\mathrm{GO}-\mathrm{CC}$ & Cell part [GO:0044464] & $4.05 E-15$ & 526 & $\begin{array}{c}\text { Atp5l, B4galt1, Dusp8, Dynll2, Exoc4, Faf1, Hint2, Hmox1, } \\
\text { Rtn2, S100al1, and so on }\end{array}$ \\
\hline $\mathrm{GO}-\mathrm{CC}$ & $\begin{array}{l}\text { Mitochondrion } \\
\text { [GO:0005739] }\end{array}$ & $7.42 E-12$ & 134 & $\begin{array}{c}\text { Atp5l, Bcs1l, Hspd1, Iars2, Ptrf, Rabl1a, Sugct, Tango2, } \\
\text { Trmt2b, Uqcc2, and so on }\end{array}$ \\
\hline GO-MF & $\begin{array}{l}\text { Catalytic activity } \\
\text { [GO:0003824] }\end{array}$ & $1.50 E-03$ & 180 & $\begin{array}{c}\text { Abhd6, Acaca, Dusp8, Ebp, Htral, Huwe1, Itpkc, Lypla1, } \\
\text { Man2al, Nek6, and so on }\end{array}$ \\
\hline REACT_188937 & Metabolism & $2.32 E-04$ & 125 & $\begin{array}{c}\text { Agpat4, Akr1c13, Cth, D2hgdh, Gstm7, Helz2, Ogn, Pank2, } \\
\text { Psma1, Suclg2, and so on }\end{array}$ \\
\hline REACT_247926 & Metabolism of proteins & 0.004417 & 58 & $\begin{array}{c}\text { Eif2s1, Eif2s3x, Eif3b, Hspd1, Igf1, Man2a1, Nfyc, Pam16, } \\
\text { Rft1, Slc30a6, and so on }\end{array}$ \\
\hline REACT_237472 & $\begin{array}{l}\text { Asparagine N-linked } \\
\text { glycosylation }\end{array}$ & 0.004713 & 19 & $\begin{array}{c}\text { Alg11, Gfpt2, Gnpnat1, Lman1, Mgat2, Rft1, Slc35a1, } \\
\text { St3gal1, St6galnac5, Uap1, and so on }\end{array}$ \\
\hline REACT_236283 & $\begin{array}{l}\text { Posttranslational protein } \\
\text { modification }\end{array}$ & 0.010703 & 27 & $\begin{array}{c}\text { Alg11, Eif5a, Galnt2, Gfpt1, Gfpt2, Man2a1, Senp5, Slc35a1, } \\
\text { St3gal1, St6galnac5, and so on }\end{array}$ \\
\hline REACT_225686 & $\begin{array}{l}\text { Autodegradation of Cdh1 } \\
\text { by Cdh1:APC/C }\end{array}$ & 0.012911 & 13 & $\begin{array}{c}\text { Cdc16, Cdc23, Cdc27, Psma1, Psma2, Psma4, Psmb4, } \\
\text { Psmb8, Psmb9, Psmc6, and so on }\end{array}$ \\
\hline REACT_219897 & $\begin{array}{l}\text { APC/C:Cdc } 20 \text { mediated } \\
\text { degradation of Securin }\end{array}$ & 0.027596 & 13 & $\begin{array}{c}\text { Cdc16, Cdc23, Cdc27, Psma1, Psma2, Psma4, Psmb4, } \\
\text { Psmb8, Psmb9, Psmc6, and so on }\end{array}$ \\
\hline
\end{tabular}

The GO-BP and GO-CC terms in the table are the top 5 ones with a higher adjusted $p$ value. DE-lncRNA, differentially time-series expressed long noncoding RNA gene; GO, Gene Ontology; BP, biological process; CC, cellular component. "REACT" terms are the pathway terms.

with human aging has also been discovered by previous studies [44, 45]. Moreover, Fbln5 was predicted to be targeted by ENSMUSG00000061510. Therefore, ENSMUSG00000086859 may also exert functions in aging via regulating the genes involved in elastic fibre formation (e.g., $F b \ln 1$ and $F b \ln 2$ ). ENSMUSG00000061510 may function in aging via regulating the expression of genes like Fbln5.

A previous study has demonstrated that aging is associated with altered cholesterol metabolism in $\mathrm{T}$ cells, causing increased cholesterol levels in lipid rafts [46]. Furthermore, cholesterol transport and lipid catabolism have been identified to be upregulated in normally aging rats [47, 48]. In the present study, CYP51 and FDPS, the positively regulated target genes of the DE-lncRNA ENSMUSG00000066057 (gene name: Gm1976), were significantly enriched in cholesterol biosynthesis. CYP51, the most evolutionarily conserved member of cytochrome P450 gene superfamily, participates in the late portion of cholesterol biosynthesis [49]. In aging peripheral nervous system and liver, CYP51 is also detected to be involved in the deregulation of cholesterol biosynthesis [50, 51]. FDPS encodes farnesyl diphosphate synthase, which is a key intermediate in cholesterol and sterol biosynthesis [52]. Previous studies have reported that FDPS is associated with bone mineral density of aging bone [53, 54]. During aging, cholesterol synthesis is reduced in human hippocampus [55]. For example, the concentration of three cholesterol precursors (lathosterol, lanosterol, and desmosterol) is significantly decreased in the hippocampus [56]. Currently, there is no experimental evidence that ENSMUSG00000066057 is involved in aging. Therefore, this DE-lncRNA may play a role in aging via the genes related to cholesterol synthesis (e.g., Cyp51 and Fdps). 
Furthermore, the DEGs that were negatively regulated by ENSMUSG00000066057 (e.g., Eif2s1) were mainly enriched in the pathways about protein metabolism along with Eif2s $3 x$ and Eif $3 b$, and several pathways about protein metabolism interacted with each other. In aging humans, the balance between muscle protein synthesis and degradation is disrupted, which leads to the loss of skeletal muscle mass [57]. All of Eif2s1, Eif2s3x, and Eif3b encode subunits of eukaryotic translation initiation factors (EIFs), which regulate protein synthesis [58]. Decreased eIF2 $\alpha$ phosphorylation has been detected in aged tissues and it is responsible for a higher level of protein phosphatase 1 and other proapoptotic proteins $[59,60]$. There is no evidence to prove the roles of Eif2s1, Eif2s3x, and Eif3b in aging so far. We speculate that the ENSMUSG00000066057 may also play critical roles in aging via regulating protein metabolism through Eif2s1. The DEGs Eif2sl, Eif2s3x, and Eif3b may also be involved in aging via protein metabolism.

Despite the aforementioned results, there were several limitations in this study. The predicted results should be confirmed by laboratory data. Furthermore, the included samples for analysis should be more. In our further studies, more samples of aging will be included to validate the expression levels and functions of the potential key lncRNAs and genes.

In conclusion, based on the gene expression data of peripheral white adipocytes taken from mice at different ages, a total of 8301 time series DEGs and 43 time series DE-lncRNAs were identified. Among them, $41 \mathrm{DE}-\ln \mathrm{RNAs}$ targeted 1880 DEGs. The DE-lncRNAs ENSMUSG00000066057, ENSMUSG00000086859, and ENSMUSG00000061510 regulated multiple DEGs. Furthermore, the DEGs positively regulated by DE-lncRNAs (e.g., ENSMUSG00000066057 and ENSMUSG00000086859) were mainly related to the functions about the development of blood vessel (e.g., Efnal and Efnb2), as well as the pathways of cholesterol biosynthesis (e.g., Cyp51 and $F d p s$ ) and elastic fibre formation (e.g., Fbln1, Fbln2, and Fbln5). Additionally, the DEGs (e.g., Eif2s1, Eif2s3x, and Eif3b) that were negatively regulated by DE-lncRNAs were correlated with the pathways about protein metabolism. These DE-lncRNAs and DEGs may be involved in aging, which provides novel information for the study of aging.

\section{Competing Interests}

All authors declare that they have no conflict of interests to state.

\section{Acknowledgments}

This study was funded by the science and technology research project from Department of Education, Heilongjiang Province (Grant no. 12531423).

\section{References}

[1] M. J. Cartwright, T. Tchkonia, and J. L. Kirkland, "Aging in adipocytes: potential impact of inherent, depot-specific mechanisms," Experimental Gerontology, vol. 42, no. 6, pp. 463-471, 2007.
[2] A. Rodriguez, D. C. Muller, M. Engelhardt, and R. Andres, "Contribution of impaired glucose tolerance in subjects with the metabolic syndrome: Baltimore Longitudinal Study of Aging," Metabolism: Clinical and Experimental, vol. 54, no. 4, pp. 542547, 2005.

[3] J. Harrington and T. Lee-Chiong, "Obesity and aging," Clinics in Chest Medicine, vol. 30, no. 3, pp. 609-614, 2009.

[4] C. B. Newgard and N. E. Sharpless, "Coming of age: molecular drivers of aging and therapeutic opportunities," Journal of Clinical Investigation, vol. 123, no. 3, pp. 946-950, 2013.

[5] M. J. Cartwright, K. Schlauch, M. E. Lenburg et al., "Aging, depot origin, and preadipocyte gene expression," The Journals of Gerontology Series A: Biological Sciences and Medical Sciences, vol. 65, no. 3, pp. 242-251, 2010.

[6] T. Tchkonia, D. E. Morbeck, T. Von Zglinicki et al., "Fat tissue, aging, and cellular senescence," Aging Cell, vol. 9, no. 5, pp. 667$684,2010$.

[7] S. A. Farr, K. A. Yamada, D. A. Butterfield et al., "Obesity and hypertriglyceridemia produce cognitive impairment," Endocrinology, vol. 149, no. 5, pp. 2628-2636, 2008.

[8] R. M. Uranga, A. J. Bruce-Keller, C. D. Morrison et al., "Intersection between metabolic dysfunction, high fat diet consumption, and brain aging," Journal of Neurochemistry, vol. 114, no. 2, pp. 344-361, 2010.

[9] C. D. Morrison, P. J. Pistell, D. K. Ingram et al., "High fat diet increases hippocampal oxidative stress and cognitive impairment in aged mice: implications for decreased Nrf2 signaling," Journal of Neurochemistry, vol. 114, no. 6, pp. 1581-1589, 2010.

[10] G. N. Landis and J. Tower, "Superoxide dismutase evolution and life span regulation," Mechanisms of Ageing and Development, vol. 126, no. 3, pp. 365-379, 2005.

[11] F. Hazane, K. Valenti, S. Sauvaigo et al., "Ageing effects on the expression of cell defence genes after UVA irradiation in human male cutaneous fibroblasts using cDNA arrays," Journal of Photochemistry and Photobiology B: Biology, vol. 79, no. 3, pp. 171-190, 2005.

[12] M. Gasparrini, D. Rivas, A. Elbaz, and G. Duque, "Differential expression of cytokines in subcutaneous and marrow fat of aging C57BL/6J mice," Experimental Gerontology, vol. 44, no. 9, pp. 613-618, 2009.

[13] K. Hotta, N. L. Bodkin, T. A. Gustafson, S. Yoshioka, H. K. Ortmeyer, and B. C. Hansen, "Age-related adipose tissue mRNA expression of ADD1/SREBP1, PPAR $\gamma$, lipoprotein lipase, and GLUT4 glucose transporter in rhesus monkeys," The Journals of Gerontology-Series A Biological Sciences and Medical Sciences, vol. 54, no. 5, pp. B183-B188, 1999.

[14] I. Grammatikakis, A. C. Panda, K. Abdelmohsen, and M. Gorospe, "Long noncoding RNAs (lncRNAs) and the molecular hallmarks of aging," Aging, vol. 6, no. 12, pp. 992-1009, 2014.

[15] J. Kim, K. M. Kim, J. H. Noh, J.-H. Yoon, K. Abdelmohsen, and M. Gorospe, "Long noncoding RNAs in diseases of aging," Biochimica et Biophysica Acta-Gene Regulatory Mechanisms, vol. 1859, no. 1, pp. 209-221, 2016.

[16] M. Guttman, I. Amit, M. Garber et al., "Chromatin signature reveals over a thousand highly conserved large non-coding RNAs in mammals," Nature, vol. 458, no. 7235, pp. 223-227, 2009.

[17] A. Fatica and I. Bozzoni, "Long non-coding RNAs: new players in cell differentiation and development," Nature Reviews Genetics, vol. 15, no. 1, pp. 7-21, 2014. 
[18] V. Bianchessi, I. Badi, M. Bertolotti et al., "The mitochondrial lncRNA ASncmtRNA-2 is induced in aging and replicative senescence in Endothelial Cells," Journal of Molecular and Cellular Cardiology, vol. 81, pp. 62-70, 2015.

[19] T.-Y. Yu, Y.-W. Kao, and J.-J. Lin, “Telomeric transcripts stimulate telomere recombination to suppress senescence in cells lacking telomerase," Proceedings of the National Academy of Sciences of the United States of America, vol. 111, no. 9, pp. 33773382, 2014.

[20] J. Khan, M. L. Bittner, Y. Chen, P. S. Meltzer, and J. M. Trent, "DNA microarray technology: the anticipated impact on the study of human disease," Biochimica et Biophysica ActaReviews on Cancer, vol. 1423, no. 2, pp. M17-M28, 1999.

[21] W. E. Bunney, B. G. Bunney, M. P. Vawter et al., "Microarray technology: a review of new strategies to discover candidate vulnerability genes in psychiatric disorders," American Journal of Psychiatry, vol. 160, no. 4, pp. 657-666, 2003.

[22] L.-F. Liu, W.-J. Shen, M. Ueno, S. Patel, and F. B. Kraemer, "Characterization of age-related gene expression profiling in bone marrow and epididymal adipocytes," BMC Genomics, vol. 12, article 212, 2011.

[23] R. A. Irizarry, B. Hobbs, F. Collin et al., "Exploration, normalization, and summaries of high density oligonucleotide array probe level data," Biostatistics, vol. 4, no. 2, pp. 249-264, 2003.

[24] L. Gautier, L. Cope, B. M. Bolstad, and R. A. Irizarry, "Affyanalysis of affymetrix genechip data at the probe level," Bioinformatics, vol. 20, no. 3, pp. 307-315, 2004.

[25] J. Harrow, A. Frankish, J. M. Gonzalez et al., "GENCODE: the reference human genome annotation for the ENCODE project," Genome Research, vol. 22, no. 9, pp. 1760-1774, 2012.

[26] M. J. Aryee, J. A. Gutiérrez-Pabello, I. Kramnik, T. Maiti, and J. Quackenbush, "An improved empirical bayes approach to estimating differential gene expression in microarray time-course data: BETR (Bayesian Estimation of Temporal Regulation)," BMC Bioinformatics, vol. 10, article 409, 2009.

[27] U. Yamaguchi, R. Nakayama, K. Honda et al., "Distinct gene expression-defined classes of gastrointestinal stromal tumor," Journal of Clinical Oncology, vol. 26, no. 25, pp. 4100-4108, 2008.

[28] G. J. Szekely and M. L. Rizzo, "Hierarchical clustering via joint between-within distances: extending Ward's minimum variance method," Journal of Classification, vol. 22, no. 2, pp. 151-183, 2005.

[29] R. S. Savage, BHC: Bayesian Hierarchical Clustering, 2010.

[30] J. Benesty, J. Chen, Y. Huang, and I. Cohen, "Pearson correlation coefficient," in Noise Reduction in Speech Processing, pp. 1-4, Springer, Berlin, Germany, 2009.

[31] M. E. Smoot, K. Ono, J. Ruscheinski, P.-L. Wang, and T. Ideker, "Cytoscape 2.8: new features for data integration and network visualization," Bioinformatics, vol. 27, no. 3, pp. 431-432, 2011.

[32] J. M. Zahn, S. Poosala, A. B. Owen et al., "AGEMAP: a gene expression database for aging in mice," PLoS Genetics, vol. 3, no. 11, pp. 2326-2337, 2007.

[33] Y.-A. Chen, L. P. Tripathi, and K. Mizuguchi, "TargetMine, an integrated data warehouse for candidate gene prioritisation and target discovery," PLoS ONE, vol. 6, no. 3, Article ID e17844, 2011.

[34] G. Van Belle, L. D. Fisher, P. J. Heagerty, and T. Lumley, Biostatistics: A Methodology for the Health Sciences, John Wiley \& Sons, New York, NY, USA, 2004.

[35] A. Larbi, C. Franceschi, D. Mazzatti, R. Solana, A. Wikby, and G. Pawelec, "Aging of the immune system as a prognostic factor for human longevity," Physiology, vol. 23, no. 2, pp. 64-74, 2008.
[36] E. Sadoun and M. J. Reed, "Impaired angiogenesis in aging is associated with alterations in vessel density, matrix composition, inflammatory response, and growth factor expression," Journal of Histochemistry and Cytochemistry, vol. 51, no. 9, pp. 1119-1130, 2003.

[37] A. U. Ferrari, A. Radaelli, and M. Centola, "Invited review: aging and the cardiovascular system," Journal of Applied Physiology, vol. 95, no. 6, pp. 2591-2597, 2003.

[38] N. Holder and R. Klein, "Eph receptors and ephrins: effectors of morphogenesis," Development, vol. 126, no. 10, pp. 2033-2044, 1999.

[39] L. W. Harries, D. Hernandez, W. Henley et al., "Human aging is characterized by focused changes in gene expression and deregulation of alternative splicing," Aging Cell, vol. 10, no. 5, pp. 868-878, 2011.

[40] C. P. Smith and J. J. Steinle, "Changes in growth factor expression in normal aging of the rat retina," Experimental Eye Research, vol. 85, no. 6, pp. 817-824, 2007.

[41] I. M. Braverman and E. Fonferko, "Studies in cutaneous aging: I. The elastic fiber network," Journal of Investigative Dermatology, vol. 78, no. 5, pp. 434-443, 1982.

[42] R. O. Hynes and A. Naba, "Overview of the matrisome-an inventory of extracellular matrix constituents and functions," Cold Spring Harbor Perspectives in Biology, vol. 4, no. 1, Article ID a004903, 2012.

[43] M. P. Jacob, "Extracellular matrix remodeling and matrix metalloproteinases in the vascular wall during aging and in pathological conditions," Biomedicine and Pharmacotherapy, vol. 57, no. 5-6, pp. 195-202, 2003.

[44] E. B. van den Akker, W. M. Passtoors, R. Jansen et al., "Metaanalysis on blood transcriptomic studies identifies consistently coexpressed protein-protein interaction modules as robust markers of human aging," Aging Cell, vol. 13, no. 2, pp. 216-225, 2014.

[45] E. B. V. d. Akker, Computational Biology in Human Aging: An Omics Data Integration Approach, Department of Medical Statistics and Bioinformatics, Faculty of Medicine, Leiden University Medical Center (LUMC), Leiden University, 2015.

[46] A. Larbi, C. Fortin, G. Dupuis, H. Berrougui, A. Khalil, and T. Fulop, "Immunomodulatory role of high-density lipoproteins: impact on immunosenescence," Age, vol. 36, no. 5, p. 9712, 2014.

[47] E. M. Blalock, K.-C. Chen, K. Sharrow et al., "Gene microarrays in hippocampal aging: statistical profiling identifies novel processes correlated with cognitive impairment," The Journal of Neuroscience, vol. 23, no. 9, pp. 3807-3819, 2003.

[48] E. M. Blalock, K.-C. Chen, A. J. Stromberg et al., "Harnessing the power of gene microarrays for the study of brain aging and Alzheimer's disease: statistical reliability and functional correlation," Ageing Research Reviews, vol. 4, no. 4, pp. 481-512, 2005.

[49] T. Režen, N. Debeljak, D. Kordiš, and D. Rozman, "New aspects on lanosterol $14 \alpha$-demethylase and cytochrome P450 evolution: lanosterol/cycloartenol diversification and lateral transfer," Journal of Molecular Evolution, vol. 59, no. 1, pp. 51$58,2004$.

[50] V. Verdier, G. Csárdi, A.-S. de Preux-Charles et al., "Aging of myelinating glial cells predominantly affects lipid metabolism and immune response pathways," Glia, vol. 60, no. 5, pp. 751760, 2012.

[51] F. Capel, G. Rolland-Valognes, C. Dacquet et al., "Analysis of sterol-regulatory element-binding protein 1c target genes 
in mouse liver during aging and high-fat diet," Journal of Nutrigenetics and Nutrigenomics, vol. 6, no. 2, pp. 107-122, 2013.

[52] D. Peschel, R. Koerting, and N. Nass, "Curcumin induces changes in expression of genes involved in cholesterol homeostasis," The Journal of Nutritional Biochemistry, vol. 18, no. 2, pp. 113-119, 2007.

[53] M. E. Levy, R. A. Parker, R. E. Ferrell, J. M. Zmuda, and S. L. Greenspan, "Farnesyl diphosphate synthase: a novel genotype association with bone mineral density in elderly women," Maturitas, vol. 57, no. 3, pp. 247-252, 2007.

[54] L. J. Dominguez, G. D. Bella, M. Belvedere, and M. Barbagallo, "Physiology of the aging bone and mechanisms of action of bisphosphonates," Biogerontology, vol. 12, no. 5, pp. 397-408, 2011.

[55] K. M. Thelen, P. Falkai, T. A. Bayer, and D. Lütjohann, "Cholesterol synthesis rate in human hippocampus declines with aging," Neuroscience Letters, vol. 403, no. 1-2, pp. 15-19, 2006.

[56] K. Smiljanic, T. Vanmierlo, A. M. Djordjevic et al., "Aging induces tissue-specific changes in cholesterol metabolism in rat brain and liver," Lipids, vol. 48, no. 11, pp. 1069-1077, 2013.

[57] R. Koopman and L. J. C. Van Loon, "Aging, exercise, and muscle protein metabolism," Journal of Applied Physiology, vol. 106, no. 6, pp. 2040-2048, 2009.

[58] B. He, M. Gross, and B. Roizman, "The $\gamma 134.5$ protein of herpes simplex virus 1 complexes with protein phosphatase $1 \alpha$ to dephosphorylate the $\alpha$ subunit of the eukaryotic translation initiation factor 2 and preclude the shutoff of protein synthesis by double-stranded RNA-activated protein kinase," Proceedings of the National Academy of Sciences of the United States of America, vol. 94, no. 3, pp. 843-848, 1997.

[59] S. G. Hussain and K. V. A. Ramaiah, "Reduced eIF2 $\alpha$ phosphorylation and increased proapoptotic proteins in aging," Biochemical and Biophysical Research Communications, vol. 355, no. 2, pp. 365-370, 2007.

[60] S. E. Wells, P. E. Hillner, R. D. Vale, and A. B. Sachs, "Circularization of mRNA by eukaryotic translation initiation factors," Molecular Cell, vol. 2, no. 1, pp. 135-140, 1998. 

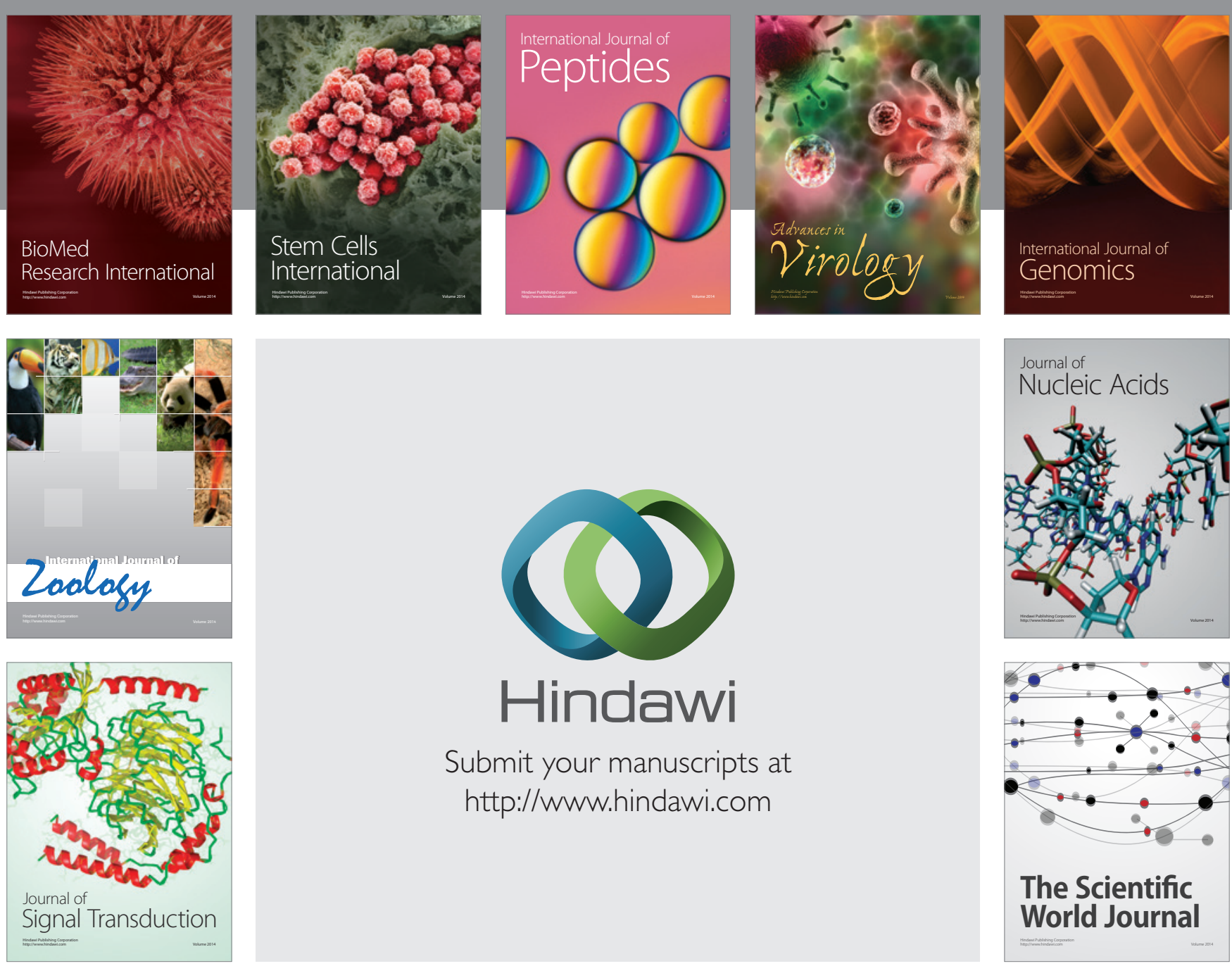

Submit your manuscripts at

http://www.hindawi.com
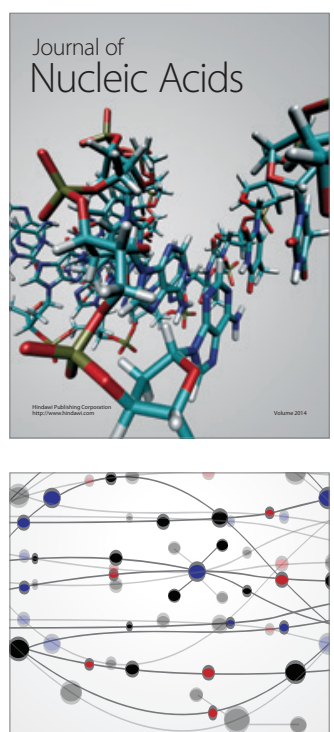

The Scientific World Journal
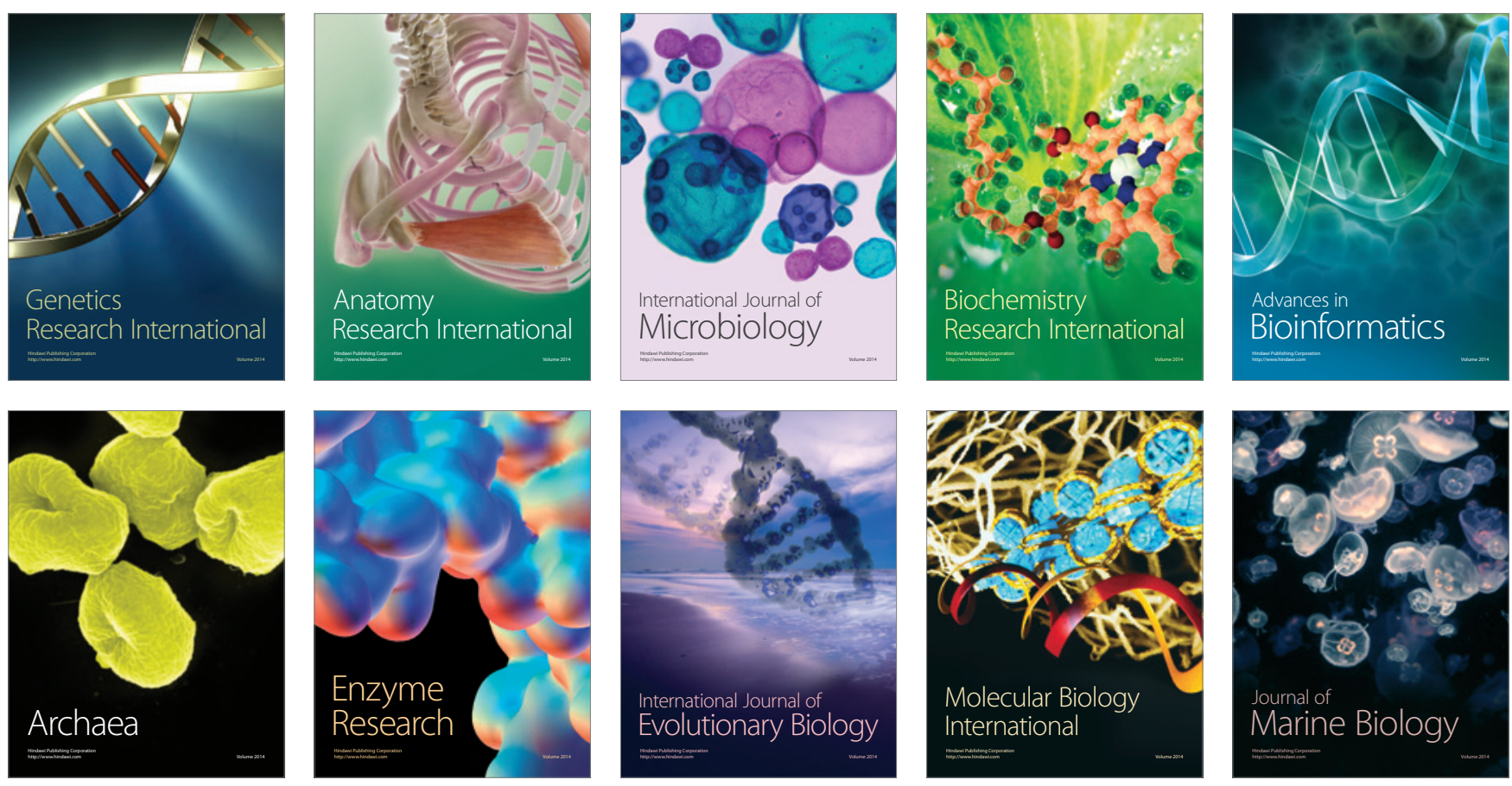